\title{
Associations between Workplace Violence, Mental Health and Physical Health among Korean Employees: The Fifth Korea Youth Working Conditions Survey (2017)
}

Hae Ran Kim ( $\sim$ rahn00@chosun.ac.kr)

\section{Research}

Keywords: Workplace violence, Mental health, Physical health, Health survey, Employee health

Posted Date: April 7th, 2020

DOI: https://doi.org/10.21203/rs.3.rs-20479/v1

License: (a) (1) This work is licensed under a Creative Commons Attribution 4.0 International License. Read Full License 


\section{Abstract}

Background This study assessed workplace violence experiences among Korean employees and its influence on mental and physical health.

Methods Using the 2017 Fifth Korea Youth Working Conditions Survey, 50,205 workers were analyzed. Experiences of workplace violence were assessed across four categories: verbal violence, unwanted sexual attention, threatening behavior, and humiliation. Mental health included anxiety, sleep problems, depressive symptoms, and physical health included hearing loss/dermatological problems, back pain, limbs myalgia, headache/eye stain, occupational injury, and overall fatigue.

Results Experience of workplace violence was more common among workers with poor health (subjectively measured), chronic disease, long weekly working hours, shift work, or jobs in the service industry. Experience of workplace violence was associated with increased anxiety, sleep problems, depression symptoms, and physical health problems.

Conclusions Prevention of workplace violence is essential to improve the health of workers.

\section{Introduction}

According to the International Labour Organization (2003)[1], workplace violence is defined as "any action, incident or behavior that departs from reasonable conduct in which a person is assaulted, threatened, harmed, or injured in the course of, or as a direct result of, his or her work." Workplace violence has become an important public health issue in many countries, and its relationship to employee health has become a trending research topic [2]. The prevalence of workplace violence varies depending on the conceptual definition, workplace culture, work characteristics, and national labor policies [3]. Decreasing trends in workplace violence have been reported in the US [4], but studies on Europe have found it is increasing [2].

Several studies have shown that workplace violence affects employee mental health. One study reported that frequent experiences of workplace violence increased the rate of anxiety and depression symptoms in the form of anger, fear, and guilt [5]. In a Korean study, employees with workplace violence experiences had a four times higher risk of sleep disorders compared to employees without such experiences [6], and another study associated such experiences with sleep problems among women [7]. Mental and physical health problems caused by workplace violence are often interconnected, and workers with workplace violence experiences have been found to maintain higher levels of tension while working, increasing their chances of poor physical health, including musculoskeletal pain [8, 9].

According to a recent Korean survey, as in Europe, workplace violence experiences among Korean employees showed a gradually increasing trend from 2005 to 2015 [10]. Despite this trajectory, few studies have examined the relationship between workplace violence and mental and physical health among Korean employees. Preventing violence in Korean workplaces will require collaborate research 
that approaches the topic from various perspectives. Therefore, this study investigated the prevalence of workplace violence among Korean employees and the association between workplace violence experiences and mental and physical health.

\section{Methods}

\section{Data source}

This study used data from the Fifth Korean Working Conditions Survey (KWCS) conducted by the Occupational Safety and Health Research Institute and distributed in 2017. The survey consisted of doorto-door interviews targeting wage workers aged 15 years and above, guaranteeing anonymity and conducted with participant consent. The survey goal was to gather information about South Korean work environments and their influence on health issues and work accidents. The goal was to foster safer and healthier workplaces.

The final sample size in the Fifth KWCS was 50,205 native employees [11]. The cooperation rate was $64.0 \%$, the contact rate was $72.1 \%$, the refusal rate was $25.3 \%$ and response rate was $44.9 \%$. These figures were calculated by the definition of the American Association for Public Opinion Research [12].

\section{Variables}

\section{General characteristics}

The general population characteristics were gender, age ( $<30,30-39,40-49$, or $\geq 50$ years), education level (elementary school, middle school, high school, undergraduate or higher, unknown, no response, or refuse), living with family (yes or no), monthly income (< US\$776, US\$776 < US\$1553, $\geq$ US\$1553; US\$1 $=1,300$ won), subjective health status (good or poor), and chronic disease (yes, or no).

\section{Work-Related Characteristics}

Work-related characteristics consisted of workplace size $(<30,30-99$, or $\geq 100$ employees), residence area (large cities, or small and middle-sized cities), length of time at current workplace $(<3,3-5,5-10$, 10 years, or missing), weekly hours worked ( $<35,35-45, \geq 46$ hours, or missing), shift work (yes or no), and occupation (managers, professionals, engineering professionals, clerks, service, sales, skilled agricultural, craft and trades, operating and assembling, elementary, armed forces, unknown, no response, or refused to respond).

\section{Experiences of Workplace Violence}

Workplace violence experiences were assessed by four criteria: verbal violence, unwanted sexual attention, threats, and humiliation. Experience was assessed using the question: "Have you ever experienced the following during the past month?" Each question was answered with "yes," or "no," or "unknown, no response, or refused to respond." Answers of unknown, no response, or refused to respond were combined with the "no," responses. 


\section{Mental Health}

Mental health included sleep problems, anxiety, and depression symptoms. Sleep problems were defined as occurring "every day," "several times a week," or "several times a month." Participants answered the question, "How often did you had the following sleep problems over the last 12 months?" Sleep problems included difficulty falling asleep, waking up often while sleeping, or waking up feeling exhausted or extremely tired. Anxiety and depression symptoms were defined as a "yes" if participants replied affirmatively to the following question, "Have you had any of the following problems during the past 12 months?"

\section{Physical Health}

Physical health included hearing loss/dermatological problems, back pain, limbs myalgia, headache/eye stain, occupational injury, and overall fatigue. Each physical health category was marked as a "yes" if identified in the participant's response to the question, "Have you had any of the following problems during the past 12 months?"

\section{Data Analysis}

A Chi-square test was used to obtain frequencies and percentages for general characteristics, workplace violence experiences, mental health, and physical health. Multiple logistic regression analyses were conducted to examine the associations between workplace violence experiences, mental health, and physical health. Finally, stratified analysis by workplace violence experiences were conducted to evaluate the association between mental and physical health. The significance level was $p=0.05$. All statistical analyses were performed using the SPSS software (version 26.0; SPSS Inc., Chicago, IL), taking into account the complex multi-stage sampling methods.

\section{Results}

\section{Experience of Workplace Violence by Subjects' General Characteristics}

Among all workers, $4.8 \%$ reported verbal abuse experiences at work, $0.9 \%$ reported unwanted sexual attention, and $0.7 \%$ reported being threatened. Humiliating episodes were reported by $3.3 \%$ of workers, and $6.6 \%$ of workers reported at least one of these experiences. The prevalence for the four categories of workplace violence was higher among workers with poor subjective health, chronic disease, long weekly working hours, shift work, and those in the service industry (Table 1). 


\begin{tabular}{|c|c|c|c|c|c|c|}
\hline Characteristics & $\begin{array}{c}\text { Number of Participants } \\
(\%)\end{array}$ & Verbal abuse & $\begin{array}{c}\text { Unwanted sexual } \\
\text { attention }\end{array}$ & Threats & Humiliation & Any experience ${ }^{a}$ \\
\hline Total & $50205(100)$ & $4.8(4.5-5.0)$ & $0.9(0.8-1.0)$ & $0.7(0.6-0.8)$ & $3.3(3.1-3.5)$ & $6.6(6.3-6.9)$ \\
\hline \multicolumn{7}{|l|}{ Gender } \\
\hline Male & 23707 (57.1) & $5.1(4.7-5.5)$ & $0.3(0.2-0.4)$ & $0.8(0.7-1.0)$ & $3.4(3.2-3.7)$ & $6.6(6.2-7.0)$ \\
\hline Female & 26498 (42.9) & $4.4(4.0-4.7)$ & $1.7(1.5-1.9)$ & $0.6(0.5-0.8)$ & $3.2(2.9-3.5)$ & $6.6(6.2-7.0)$ \\
\hline \multicolumn{7}{|l|}{ Age (yr) } \\
\hline$<30$ & 4457 (14.8) & $6.0(5.2-6.9)$ & $1.6(1.2-2.1)$ & $1.1(0.7-1.6)$ & $3.6(3.0-4.3)$ & $8.4(7.5-9.5)$ \\
\hline 30-39 & 8497 (21.1) & $4.8(4.2-5.4)$ & $1.2(0.9-1.5)$ & $0.6(0.5-0.8)$ & $3.3(2.8-3.8)$ & $6.8(6.1-7.5)$ \\
\hline $40-49$ & $11795(24.5)$ & $4.6(4.1-5.1)$ & $0.8(0.7-1.1)$ & $0.7(0.6-1.0)$ & $3.3(2.9-3.7)$ & $6.3(5.7-6.8)$ \\
\hline$\geq 50$ & 25456 (39.6) & $4.5(4.1-4.8)$ & $0.6(0.5-0.7)$ & $0.7(0.5-0.8)$ & $3.3(3.0-3.6)$ & $6.0(5.6-6.4)$ \\
\hline \multicolumn{7}{|l|}{ Education level } \\
\hline Elementary school & $5205(5.4)$ & $2.6(2.0-3.4)$ & $0.1(0.0-0.2)$ & $0.2(0.1-0.5)$ & $2.1(1.6-2.9)$ & $3.8(3.0-4.7)$ \\
\hline Middle school & $4546(6.7)$ & $5.6(4.7-6.5)$ & $0.3(0.2-0.5)$ & $0.5(0.3-0.7)$ & $3.6(2.9-4.3)$ & $6.8(5.9-7.9)$ \\
\hline High school & $19156(34.7)$ & $5.9(5.4-6.4)$ & $1.1(1.0-1.4)$ & $0.7(0.5-0.9)$ & $4.1(3.8-4.5)$ & $8.0(7.5-8.5)$ \\
\hline College or higher & $21246(53.2)$ & $4.2(3.9-4.6)$ & $0.9(0.8-1.1)$ & $0.9(0.7-1.0)$ & $2.9(2.6-3.2)$ & $6.0(5.6-6.4)$ \\
\hline Unknown/no response/refuse & $52(0.1)$ & $1.3(0.2-8.8)$ & - & - & - & $1.3(0.2-8.8)$ \\
\hline \multicolumn{7}{|l|}{ Living with family } \\
\hline Yes & $40723(92.9)$ & $4.8(4.5-5.1)$ & $0.9(0.8-1.0)$ & $0.8(0.6-0.9)$ & $3.3(3.1-3.6)$ & $6.6(6.3-6.9)$ \\
\hline No & $9482(7.1)$ & $4.6(4.0-5.1)$ & $1.1(0.9-1.4)$ & $0.5(0.3-0.7)$ & $3.0(2.6-3.5)$ & $6.7(6.0-7.4)$ \\
\hline \multicolumn{7}{|l|}{ Monthly income } \\
\hline$\geq 1553$ & $1230(2.6)$ & $5.3(3.8-7.4)$ & $0.7(0.2-1.9)$ & $1.4(0.7-2.9)$ & $2.6(1.6-4.1)$ & $6.2(4.6-8.4)$ \\
\hline $776-<1553$ & $1243(2.1)$ & $3.6(2.2-6.0)$ & $0.9(0.4-2.0)$ & $0.7(0.3-1.9)$ & $3.2(1.9-5.3)$ & $5.3(3.5-7.8)$ \\
\hline$<776$ & $47732(95.3)$ & $4.8(4.5-5.1)$ & $0.9(0.8-1.0)$ & $0.7(0.6-0.8)$ & $3.3(3.1-3.6)$ & $6.6(6.3-6.9)$ \\
\hline \multicolumn{7}{|l|}{ Subjective health status } \\
\hline Good & 33058 (72.6) & $4.0(3.8-4.3)$ & $0.8(0.7-1.0)$ & $0.6(0.5-0.7)$ & $2.8(2.6-3.1)$ & $5.8(5.4-6.1)$ \\
\hline Poor & $17147(27.4)$ & $6.7(6.2-7.3)$ & $1.1(0.9-1.3)$ & $1.1(0.9-1.3)$ & $4.6(4.2-5.1)$ & $8.8(8.2-9.4)$ \\
\hline \multicolumn{7}{|l|}{ Chronic disease } \\
\hline No & $46699(95.1)$ & $4.5(4.3-4.8)$ & $0.9(0.8-1.0)$ & $0.7(0.6-0.8)$ & $3.1(2.9-3.3)$ & $6.2(6.0-6.6)$ \\
\hline Yes & $3506(4.9)$ & $9.8(8.4-11.4)$ & $1.2(0.8-1.8)$ & $1.4(0.9-2.2)$ & $7.9(6.7-9.3)$ & $13.3(11.7-15.1)$ \\
\hline \multicolumn{7}{|l|}{ Workplace size } \\
\hline$<30$ workers & $47581(92.8)$ & $4.7(4.4-5.0)$ & $0.9(0.8-1.0)$ & $0.7(0.6-0.8)$ & $3.2(3.0-3.4)$ & $6.5(6.2-6.8)$ \\
\hline 30-99 workers & $1257(3.1)$ & $6.4(5.0-8.3)$ & $1.8(1.1-2.9)$ & $2.2(1.4-3.6)$ & $5.3(4.0-6.9)$ & $9.3(7.5-11.4)$ \\
\hline$\geq 100$ workers & $1367(4.2)$ & $5.5(4.2-7.3)$ & $0.3(0.2-0.8)$ & $1.2(0.6-2.1)$ & $4.6(3.3-6.2)$ & $7.3(5.8-9.1)$ \\
\hline \multicolumn{7}{|l|}{ Area of residence } \\
\hline Large cities & $22532(46.4)$ & $5.1(4.7-5.4)$ & $0.8(0.7-0.9)$ & $0.7(0.6-0.8)$ & $3.2(2.9-3.5)$ & $6.8(6.4-7.2)$ \\
\hline Small and middle-sized cities & $27673(53.6)$ & $4.5(4.2-4.9)$ & $1.0(0.8-1.2)$ & $0.8(0.6-0.9)$ & $3.4(3.2-3.7)$ & $6.4(6.0-6.8)$ \\
\hline \multicolumn{7}{|l|}{ Working period at present } \\
\hline \multicolumn{7}{|l|}{ workplace (yr) } \\
\hline$<3$ & $11195(24.6)$ & $5.7(5.1-6.3)$ & $1.1(0.9-1.4)$ & $0.8(0.6-1.1)$ & $3.3(2.9-3.8)$ & $7.7(7.1-8.4)$ \\
\hline 3-5 & $12952(26.6)$ & $5.2(4.7-5.7)$ & $1.3(1.0-1.5)$ & $0.7(0.6-1.0)$ & $3.7(3.3-4.2)$ & $7.3(6.7-7.9)$ \\
\hline $5-10$ & $9875(20.6)$ & $4.9(4.4-5.5)$ & $0.9(0.7-1.2)$ & $0.7(0.5-1.0)$ & $3.7(3.2-4.2)$ & $6.9(6.3-7.6)$ \\
\hline$>10$ & $15094(26.2)$ & $3.4(3.1-3.9)$ & $0.3(0.2-0.5)$ & $0.7(0.5-0.9)$ & $2.6(2.3-3.0)$ & $4.6(4.2-5.1)$ \\
\hline Missing & 1089 (1.9) & $3.6(2.5-5.1)$ & $1.0(0.5-2.0)$ & $0.6(0.2-1.6)$ & $4.0(2.7-5.9)$ & $6.7(5.0-8.8)$ \\
\hline \multicolumn{7}{|l|}{ Weekly working hours } \\
\hline$<35$ & 7245 (11.5) & $3.4(2.8-4.0)$ & $0.8(0.6-1.1)$ & $0.3(0.2-0.4)$ & $2.2(1.8-2.7)$ & $4.8(4.2-5.6)$ \\
\hline $35-45$ & $20771(49.3)$ & $3.8(3.5-4.2)$ & $0.8(0.7-1.0)$ & $0.7(0.5-0.8)$ & $2.7(2.4-3.0)$ & $5.4(5.0-5.8)$ \\
\hline$\geq 46$ & $21943(38.8)$ & $6.4(5.9-6.8)$ & $1.0(0.9-1.2)$ & $0.9(0.8-1.2)$ & $4.5(4.1-4.9)$ & $8.6(8.1-9.2)$ \\
\hline Missing & $246(0.4)$ & $7.6(3.9-14.3)$ & $1.0(0.3-3.7)$ & $1.3(0.4-3.7)$ & $5.1(2.4-10.5)$ & $8.5(4.6-15.1)$ \\
\hline \multicolumn{7}{|l|}{ Shift work } \\
\hline No & $45953(90.3)$ & $4.1(3.9-4.4)$ & $0.9(0.8-1.0)$ & $0.5(0.4-0.6)$ & $2.8(2.6-3.0)$ & $5.8(5.5-6.1)$ \\
\hline \multirow[t]{2}{*}{ Yes } & $4252(9.7)$ & $11.1(9.9-$ & $1.3(1.0-1.8)$ & $2.9(2.2-3.7)$ & $7.9(6.9-9.0)$ & $14.3(13.0-15.8)$ \\
\hline & & 12.4) & & & & \\
\hline \multicolumn{7}{|l|}{ Occupation } \\
\hline Managers & $1216(3.3)$ & $3.1(2.1-4.4)$ & $0.7(0.3-1.7)$ & $0.8(0.3-1.7)$ & $2.3(1.5-3.6)$ & $4.3(3.1-5.9)$ \\
\hline Professionals & $3403(8.7)$ & $3.0(2.3-3.8)$ & $0.7(0.4-1.0)$ & $0.9(0.5-1.5)$ & $2.2(1.7-3.0)$ & $4.4(3.6-5.4)$ \\
\hline Engineering professionals & $1834(5.4)$ & $3.2(2.3-4.4)$ & $0.6(0.3-1.1)$ & $0.8(0.4-1.4)$ & $2.4(1.6-3.4)$ & $4.7(3.6-6.0)$ \\
\hline Clerks & $7182(20.8)$ & $3.3(2.8-3.9)$ & $1.0(0.7-1.3)$ & $0.6(0.4-0.9)$ & $2.0(1.6-2.4)$ & $4.9(4.3-5.6)$ \\
\hline Service & $9104(15.6)$ & $7.7(7.0-8.6)$ & $2.0(1.6-2.4)$ & $1.4(1.1-1.8)$ & $5.1(4.5-5.8)$ & $10.2(9.3-11.1)$ \\
\hline Sales & $10168(14.4)$ & $5.4(4.8-6.1)$ & $1.3(1.1-1.6)$ & $0.6(0.4-0.8)$ & $4.3(3.8-4.9)$ & $8.1(7.4-8.9)$ \\
\hline
\end{tabular}

Page 5/12 


\section{Associations between Workplace Violence Experiences and Mental health}

In terms of mental health problems among workers, the prevalence of anxiety was $3.1 \%$, sleep problems were $20.5 \%$, and of depression symptoms were $2.4 \%$. Compared to workers with no violence experience, workers with verbal abuse experiences were more likely to report anxiety (adjusted odds ratio $(\mathrm{aOR})=$ $3.95,95 \% \mathrm{Cl}=3.21-4.85)$, sleep problems $(\mathrm{aOR}=1.55,95 \% \mathrm{Cl}=1.37-1.76)$, and depression symptoms $(\mathrm{aOR}=2.54,95 \% \mathrm{Cl}=1.89-3.42)$. Likewise, unwanted sexual attention, threats, and humiliation were associated with increased anxiety, sleep problems, and depression symptoms (Table 2).

Table 2 Associations between experience of workplace violence and mental health.

\begin{tabular}{lcccccc}
\hline \multirow{2}{*}{ Variables } & \multicolumn{2}{c}{ Anxiety } & \multicolumn{2}{c}{ Sleep problem } & \multicolumn{2}{c}{ Depressive symptoms ${ }^{\mathrm{b}}$} \\
\cline { 2 - 7 } & $\%$ & OR (95\%CI) ${ }^{\mathrm{a}}$ & $\%$ & OR (95\%CI) & ${ }^{\mathrm{O}}$ & OR $^{(95 \% \mathrm{CI})}{ }^{\mathrm{b}}$ \\
\hline Total & 3.1 & & 20.5 & & 2.4 & \\
\hline Verbal abuse & 11.5 & $3.95(3.21-4.85)$ & 31.2 & $1.55(1.37-1.76)$ & 9.6 & $2.54(1.89-3.42)$ \\
Unwanted sexual attention & 11.8 & $4.03(2.71-6.00)$ & 30.4 & $1.55(1.21-1.99)$ & 11.4 & $2.85(1.58-5.13)$ \\
Threats & 21.0 & $7.22(4.91-10.64)$ & 47.3 & $3.03(2.24-4.10)$ & 17.8 & $2.93(1.66-5.17)$ \\
Humiliation & 13.0 & $4.22(3.39-5.26)$ & 38.0 & $2.11(1.84-2.43)$ & 11.8 & $2.95(2.07-4.19)$ \\
Any experience & 10.5 & $3.70(3.07-4.46)$ & 31.2 & $1.59(1.44-1.78)$ & 9.1 & $2.76(2.10-3.62)$ \\
\hline
\end{tabular}

CI, confidence interval; OR, odds ratio.

${ }^{a}$ Adjusted for gender, age, education, living with family, monthly income, subjective health status, chronic disease, workplace size, area of

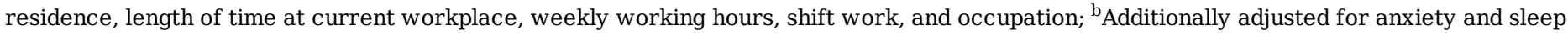
problems.

\section{Associations between Experience of Workplace Violence and Physical health}

In terms of workers' physical health problems, the prevalence of hearing loss/dermatological problems was $1.9 \%$, back pain was $11.5 \%$, limbs myalgia was $27.2 \%$, headache/eye strain was $13.4 \%$, occupational injury was $1.6 \%$, and of overall fatigue was $23.4 \%$. Compared to workers without workplace violence 
experiences, workers with experiences of verbal abuse, unwanted sexual attention, threats, and humiliation were more likely to report hearing loss/dermatological problems, back pain, limbs myalgia, headache/eye strain, occupational injury, and overall fatigue (Table 3).

Table 3 Associations between experience of workplace violence and physical health.

\begin{tabular}{|c|c|c|c|c|c|c|c|c|c|c|c|c|}
\hline \multirow[t]{2}{*}{ Variables } & \multicolumn{2}{|c|}{$\begin{array}{c}\text { Hearing } \\
\text { loss } / \text { dermatologic } \\
\text { problem }\end{array}$} & \multicolumn{2}{|c|}{ Back Pain } & \multicolumn{2}{|c|}{ Limbs myalgia } & \multicolumn{2}{|c|}{$\begin{array}{l}\text { Headache/eye } \\
\text { stain }\end{array}$} & \multicolumn{2}{|c|}{$\begin{array}{l}\text { Occupational } \\
\text { injury }\end{array}$} & \multicolumn{2}{|c|}{ Overall fatigue } \\
\hline & $\%$ & OR $(95 \% C I)^{a}$ & $\%$ & $\begin{array}{c}\text { OR } \\
(95 \% \mathrm{CI})^{\mathrm{a}} \\
\end{array}$ & $\%$ & $\begin{array}{c}\text { OR } \\
(95 \% \mathrm{CI})^{\mathrm{a}} \\
\end{array}$ & $\%$ & $\begin{array}{c}\text { OR } \\
(95 \% \mathrm{CI})^{\mathrm{a}} \\
\end{array}$ & $\%$ & $\begin{array}{c}\text { OR } \\
(95 \% C I)^{a} \\
\end{array}$ & $\%$ & $\begin{array}{c}\text { OR } \\
(95 \% \mathrm{CI})^{\mathrm{a}} \\
\end{array}$ \\
\hline Total & 1.9 & & 11.5 & & 27.2 & & 13.4 & & 1.6 & & 23.4 & \\
\hline Verbal abuse & 3.9 & $1.83(1.30-2.56)$ & 24.7 & $\begin{array}{c}2.65(2.29- \\
3.08)\end{array}$ & 47.4 & $\begin{array}{l}2.47(2.17- \\
2.81)\end{array}$ & 27.2 & $\begin{array}{l}2.43 \\
(2.12- \\
2.78)\end{array}$ & 3.7 & $\begin{array}{l}1.96 \\
(1.43- \\
2.69)\end{array}$ & 45.9 & $\begin{array}{l}2.65 \\
(2.34- \\
3.01)\end{array}$ \\
\hline $\begin{array}{l}\text { Unwanted sexual } \\
\text { attention }\end{array}$ & 3.9 & $2.60(1.30-5.20)$ & 11.4 & $\begin{array}{c}2.24(1.61- \\
3.13)\end{array}$ & 36.5 & $\begin{array}{l}1.73(1.31- \\
2.29)\end{array}$ & 22.0 & $\begin{array}{l}1.92 \\
(1.44- \\
2.56)\end{array}$ & 4.6 & $\begin{array}{l}3.82 \\
(2.14- \\
6.82)\end{array}$ & 33.0 & $\begin{array}{l}1.78 \\
(1.34- \\
2.36)\end{array}$ \\
\hline Threats & 7.8 & $3.91(2.16-7.10)$ & 11.3 & $\begin{array}{c}3.62(2.60- \\
5.04)\end{array}$ & 52.4 & $\begin{array}{c}3.23(2.35- \\
4.46)\end{array}$ & 32.8 & $\begin{array}{c}2.69 \\
(1.97- \\
3.68)\end{array}$ & 5.0 & $\begin{array}{l}2.44 \\
(1.32- \\
4.50)\end{array}$ & 50.5 & $\begin{array}{l}3.09 \\
(2.26- \\
4.24)\end{array}$ \\
\hline Humiliation & 4.9 & $2.84(2.07-3.90)$ & 10.9 & $\begin{array}{c}3.35(2.86- \\
3.93)\end{array}$ & 51.7 & $\begin{array}{l}3.18(2.76- \\
3.65)\end{array}$ & 30.5 & $\begin{array}{c}3.09 \\
(2.67- \\
3.57)\end{array}$ & 3.7 & $\begin{array}{c}1.86 \\
(1.28- \\
2.69)\end{array}$ & 51.9 & $\begin{array}{c}3.41 \\
(2.96- \\
3.94)\end{array}$ \\
\hline Any experience & 3.9 & $2.23(1.59-3.12)$ & 10.6 & $\begin{array}{c}3.00(2.54- \\
3.55)\end{array}$ & 45.8 & $\begin{array}{c}2.88(2.49- \\
3.34)\end{array}$ & 25.7 & $\begin{array}{r}2.85 \\
(2.44- \\
3.31) \\
\end{array}$ & 3.8 & $\begin{array}{r}2.25 \\
(1.71- \\
2.97) \\
\end{array}$ & 43.4 & $\begin{array}{l}2.52 \\
(2.26- \\
2.81) \\
\end{array}$ \\
\hline
\end{tabular}

CI, confidence interval; OR, odds ratio.

${ }^{a}$ Adjusted for gender, age, education, living with family, monthly income, subjective health status, chronic disease, workplace size, area of residence, length of time at current workplace, weekly working hours, shift work, and occupation.

\section{Discussion}

This study investigated employees' workplace violence experiences and the influence of workplace violence on mental and physical health among Koreans. In this study, $6.6 \%$ experienced at least one episode of verbal abuse, unwanted sexual attention, threats, or humiliation. Workplace violence experiences were associated with increased physical and mental health problems.

Previous studies showed the prevalence of workplace violence in difference countries varies depending on the environment, experience, and measurement. In a large study of US adults, less than $8.1 \%$ of workers reported harassment in the workplace over a 12-month period, but those that did suffered from pain disorders and psychosocial distress [8]. In a national survey of the US labor force, over $60 \%$ of respondents experienced generalized workplace harassment, including verbal aggression, disrespectful behavior, isolation/exclusion, threats/bribes, and physical aggression [13]. In a 2018 survey about workplace violence administered to health management experts in China, the overall prevalence was $62.4 \%$ [14]. In a representative survey of working environments in Denmark, around $12 \%$ of respondents were exposed to workplace harassment [3], and 2.2\% reported unwanted sexual attention [15]. This was similar to the results of the 2016 KWCS, which reported the prevalence of workplace violence in South 
Korea as $6.0 \%$ [6]. Discrepancies in the reported prevalence can be explained by differences in the conceptualization and definition of workplace violence, as well as diverse cultural attitudes toward workplace violence. In the present study, physical violence, sexual harassment, and exclusion/harassment were excluded from the analysis of workplace violence. Within the Korean workforce, fear of retaliation and the belief that reporting violence will not change practices, meant that workers were hesitant to admit their level of workplace violence [16]. Therefore, it is possible that the frequency of experiences could have been underestimated due to a lack of information, but further research is needed to account for this possibility.

Of the four categories of violence, verbal abuse was reported most frequently. In a nationwide, crosssectional study of female Korean workers, $9.2 \%$ of the participants experienced verbal violence [17]. Another study on Korean employees also reported that verbal violence was most frequent, with a prevalence of $4.8 \%$ [18]. Verbal abuse occurs more frequently because it is rarely recorded or reported to human resources [19]. Encouraging verbal abuse reporting and providing thorough feedback could help to prevent overall workplace violence [20].

Similar to previous studies, we observed differences in workplace violence experiences depending on the population's general characteristics. Participants were more likely to report workplace violence if they had poor health, chronic disease, longer weekly working hours, performed shift work, or were employed in the service industry. Also, negative moods such as fear or stress, resulting from workplace violence can lead to an increased risk of cardiovascular disease [21] or exacerbate chronic health problems such as depression $[8,22]$. Longer working hours and shift work were occupational risk factors for violence among Korean workers [18]. It is possible that excessively long shifts due to staff shortages or dilapidated working environments could lead to a higher risk of violence between workers [23]. Service occupations impose a higher burden of emotional labor and emotional burnout, and so even small communication issues can expose workers to violence [17, 24]. Appropriate workload intensity and emotion management training could help to prevent workplace violence and ameliorate chronic diseases.

Like other studies $[11,13,17]$, workplace violence experiences among Korean workers were associated with poorer mental and physical health. Experiencing verbal or physical workplace violence can elevate stress hormone levels, leading directly to increased anxiety, depressive moods [25], and sleep disorders [6]. Workplace violence can cause workers tension or strain, which can increase the risk of musculoskeletal disease [26]. One previous study reported workers with physical health problems can exhibit increased mental stress due to discrimination, fear of losing trust, and fear of criticism [27]. Physical health issues owing to workplace violence can make workers more vulnerable to mental illness, and workers with mental health issues can be exposed to greater physical health risks.

The present study had several limitations. First, mental and physical health are also associated with occupation-specific characteristics, work environments, and supervision factors. In addition, health outcomes were assessed using a self-reported questionnaire, and actual diagnoses and risk groups could not be inspected. Further studies are needed to account for this data. Second, because this was a cross- 
sectional study, we were unable to analyze causal relationships between variables. Longitudinal research is needed to investigate causal relationships for the purpose of preventing mental and physical health problems caused by workplace violence, as well as managing health problems that have already occurred.

\section{Conclusions}

Using nationally representative data, this study demonstrated that workplace violence experiences influenced mental and physical health among Korean workers. Since consistent workplace violence poses a risk to employee's overall health, it is necessary to implement and maintain workplace policies and educational programs to prevent workplace violence.

\section{Abbreviations}

KWCS, Korean Working Conditions Survey

\section{Declarations}

\section{Ethics approval and consent to participate}

Not applicable.

\section{Consent for publication}

Not applicable.

\section{Availability of data and materials}

This study used publicly accessible information from the Korea Occupational Safety and Health Agency website (http://www.kosha.or.kr/kosha/data/primitiveData.do).

\section{Competing interests}

The authors declare that they have no competing interests.

\section{Funding}

Not applicable.

\section{Author information}

\section{Affiliations}

Department of Nursing, College of Medicine, Chosun University, 309 Pilmun-daero, Dong-gu, Gwangju 61452 , Republic of Korea. 
Contribution

All work on this manuscript was done by HRK. No other persons have contributed to or reviewed this manuscript prior to submission. The author read and approved the final manuscript.

Corresponding author

Correspondence to Hae Ran Kim(rahn00@chosun.ac.kr)

\section{Acknowledgements}

Not applicable.

\section{References}

1. International Labour Organization Geneva. 2003. Code of practice on workplace violence in services sectors and measures to combat this phenomenon. [Accessed 2 March 2020] Available from URL: https://www.ilo.org/safework/info/standards-and-instruments/codes/WCMS_107705/langen/index.htm

2. Bossche S, Taris T, Houtman I, Smulders P, Kompier M. Workplace violence and the changing nature of work in Europe: Trends and risk groups. European Journal of Work and Organizational Psychology. 2013;22:588-600.

3. Török E, Hansen ÅM, Grynderup MB, Garde AH, Høgh A, Nabe-Nielsen K. The association between workplace bullying and depressive symptoms: the role of the perpetrator. BMC Public Health. 2016;16:993.

4. Foley M, Rauser E. Evaluating progress in reducing workplace violence: Trends in Washington State workers' compensation claims rates, 1997-2007. Work. 2012;42(1):67-81.

5. Lanctôt N, Guay S. The aftermath of workplace violence among healthcare workers: A systematic literature review of the consequences. Aggress Violent Behav. 2014;19(5):492-501.

6. Yoo TJ, Ye BJ, Kim JI, Park SW. Relationship of workplace violence and perpetrators on sleep disturbance-data from the 4th Korean working conditions survey. Ann Occup Environ Med. 2016;28(1):59.

7. Lallukka T, Rahkonen O, Lahelma E. Workplace bullying and subsequent sleep problems - the Helsinki Health Study. Scand J Work Environ Health. 2011(3):204-12.

8. Khubchandani J, Price JH. Workplace harassment and morbidity among US adults: Results from the National Health Interview Survey. J Community Health. 2015;40(3):555-63.

9. Sivertsen $\mathrm{H}$, Lillefjell M, Espnes GA. The relationship between health promoting resources and work participation in a sample reporting musculoskeletal pain from the Nord-Trøndelag Health Study, HUNT 3, Norway. BMC Musculoskelet Disord. 2013;14:100. 
10. Kim GR. Workplace mistreatment in Korea and Europe - Comparison study of discrimination, sexual harassment and violence: Seoul National University; 2017.

11. Kang DK, Yang JW, Choi WJ, Ham SH, Kang SK, Lee WH. Anxiety, depression and sleep disturbance among customer-facing workers. J Korean Med Sci. 2019;34(48):e313.

12. Ahn JH, Cho SS, Kim HR, Myong JP, Kang MY. Comparison of work environment and occupational injury in direct and indirect employment in Korea and Europe. Ann Occup Environ Med. 2019;31:e24.

13. Rospenda KM, Richman JA, Shannon CA. Prevalence and mental health correlates of harassment and discrimination in the workplace: results from a national study. J Interpers Violence. 2009;24(5):819-43.

14. Lu L, Dong M, Wang SB, Zhang L, Ng CH, Ungvari GS, et al. Prevalence of workplace violence against health-care professionals in China: A comprehensive meta-analysis of observational surveys. Trauma Violence Abuse. 2018;1524838018774429.

15. Hogh A, Conway PM, Clausen T, Madsen IEH, Burr H. Unwanted sexual attention at work and longterm sickness absence: a follow-up register-based study. BMC Public Health. 2016;16:678.

16. Ramacciati N, Bambi S, Rasero L. Workplace violence against nurses in Korea and its impact on professional quality of life and turnover intention. J Nurs Manag. 2018;26(3):332-3.

17. Kim EJ, Yoon JY. Effects of emotional labor and workplace violence on physical and mental health outcomes among female workers: The 4th Korean Working Conditions Survey. Korean Journal of Occupational Health Nursing. 2017;26(3):184-96.

18. Lee HE, Kim HR, Park JS. Work-related risk factors for workplace violence among Korean employees. J Occup Health. 2014;56(1):12-20.

19. Zoleo M, Della Rocca F, Tedeschi F, Zucchetto M, Maddalena G, Vettore G. Violence against health workers: findings from three emergency departments in the teaching hospital of Padua, Italy. Intern Emerg Med. 2020.

20. Fisekovic Kremic MB, Terzic-Supic ZJ, Santric-Milicevic MM, Trajkovic GZ. Encouraging employees to report verbal violence in primary health care in Serbia: A cross-sectional study. Zdr Varst. 2016;56(1):11-7.

21. Xu T, Magnusson Hanson LL, Lange T, Starkopf L, Westerlund H, Madsen IEH, et al. Workplace bullying and violence as risk factors for type 2 diabetes: a multicohort study and meta-analysis. Diabetologia. 2018;61(1):75-83.

22. Jung PK, Won J-U, Roh J, Lee J-H, Seok H, Lee W, et al. Workplace Violence Experienced by Substitute (Daeri) Drivers and Its Relationship to Depression in Korea. J Korean Med Sci. 2015;30(12):1748-53.

23. Sun S, Gerberich SG, Ryan AD. The relationship between shiftwork and violence against nurses: $A$ case control study. Workplace Health Saf. 2017;65(12):603-11.

24. Eyasu N, Taa B. Effects of workplace violence on women's psychosocial functioning in Ethiopia: Emotional demand and social relations at civil service sectors in focus. J Interpers Violence. 2019;0886260519888634. 
25. Isaksson J, Comasco E, Aslund C, Rehn M, Tuvblad C, Andershed H, et al. Associations between the FKBP5 haplotype, exposure to violence and anxiety in females. Psychoneuroendocrinology. 2016;72:196-204.

26. Choi SJ, Yi YJ, Kim JY. Exposure to adverse social behavior in the workplace and sickness presenteeism among Korean workers: The mediating effects of musculoskeletal disorders. Int $\mathrm{J}$ Environ Res Public Health. 2018;15(10):2198.

27. Ree E, Johnsen TL, Harris A, Malterud K. Workplace inclusion of employees with back pain and mental health problems: A focus group study about employees' experiences. Scand J Public Health. 2019;47(3):326-33. 